vom 3. Juli 1985 - VIII ZR 102/84 (WM 1985, 906) ein. Denn in dem entschiedenen Fall waren Absprachen des Leasingnehmers mit dem Lieferanten über ein Rücktrittsrecht wegen fehlerhaften Verhaltens des Lieferanten bei den Vertragsverhandlungen nicht zum Inhalt des Leasingvertrages geworden. Im vorliegenden Fall ist aber die Vereinbarung mit dem Lieferanten schriftlich festgehalten (Schreiben des Lieferanten vom 24. Februar 1982). Danach hat die Beklagte selbst dafür gesorgt, daß die Zusatzvereinbarung zum Inhalt des Leasingvertrages wurde, indem sie die Zusatzklausel in den Leasingantrag einfügte. Sie hatte es in der Hand, die Nebenabsprachen zum Bestandteil des Leasingvertrages zu machen, und hat davon Gebrauch gemacht. Einen Hinweis darauf, daß die Aufhebung des bestehenden Leasingvertrages von der Verwirklichung des Umtausches gegen eine neue Anlage durch den Lieferanten abhängig war, brauchte die Klägerin der Beklagten als einem kaufmännischen Vertragspartner nicht zu geben.

2. Da die Beklagte die Leasingraten vertragswidrig ab 1. Januar 1983 nicht mehr zahlte, war die Klägerin berechtigt, durch die außerordentliche Kündigung vom 25. Oktober 1983 ihrerseits das Leasingverhältnis mit der Beklagten zu beenden.

Nach dem Urteil des Bundesgerichtshofs vom 24. April 1985 - VIII ZR 65/84 (WM 1985, 638, 641) hat der Leasinggeber nach berechtigter fristloser Kündigung gegen den Leasingnehmer einen sofort fälligen Schadensersatzanspruch auf Zahlung der restlichen für die kalkulierte Vertragsdaner vorgesehenen Leasingraten. Daran abzusetzen sind die Vorteile aus der vorzeitigen Erlangung des sonst pro rata temporis fälligen Betrages (also Abzinsung) und aus der vorzeitigen Rückerlangung der Leasingsache (Wiederverkaufserlös).

Die Berechnung der Klägerin vernachlässigt die für sie günstigere Regelung in $\S 9$ des Formularvertrages und hat einen gesetzlichen, den Anforderungen der Rechtsprechung des BGH entsprechenden Schadensersatzanspruch zum Inhalt.
Die Abzinsung ist mit 9,75\% höher als vertraglich vorgesehen. Der Wiederverkaufserlös, den die Klägerin erzielt hat, bewegt sich in dem Rahmen, der nach dem Gutachten des Sachverständigen Dr. X. realistisch ist. Wenn der Veräußerungswert im November 1983 schlechtestenfalls $1000,-$ DM war, erscheinen $800,-$ DM im Mai 1985 nicht zu niedrig. Daß vorher schon eine - eventuell auch bessere - Veräußerungsmöglichkeit bestand, ist nicht dargetan.

Die Beklagte berücksichtigt bei ihren Angriffen auf das Gutachten nicht, daß es nicht darum geht, den Preis zıl ermitteln, den ein zufällig existierender Interessent, wenn er ausfindig gemacht werden könnte, als Enderwerber der Anlage einem Händler bezahlen würde. Vielmehr muß von den Verkaufsmöglichkeiten einer Leasinggesellschaft ausgegangen werden. Diese sind in der Regel nur gegenüber Händlern gegeben. Bei Händlern besteht jedoch - wie der Sachverständige in seiner mündlichen Anhörung überzeugend ausgeführt hat - hinsichtlich eines gebrauchten und nicht der neuesten Serie entstammenden Geräts der vorliegenden Art ein Marktinteresse im allgemeinen nur zur Ersatzteilbeschaffung. Die Anlagen werden gekauft, um deraus Verschleißteile auszubauen, die zur Reparatur von noch in Betrieb befindlichen Geräten bei Kunden benötigt werden.

Die Beklagte hat auch keine konkrete bessere VeräuBerungsmöglichkeit für das Gerät vortragen können.

$\mathrm{Ob}$ die Beklagte einen Anspruch auf Anrechnung des vollen Nettoerlöses hätte (vgl. BGH NJW 1985, 2257), kann hier dahinstehen, weil die Klägerin jedenfalls den gesamten Nettoerlös zugunsten der Beklagten in Abzug bringt.

3. Die Aufreehnung der Beklagten in der Berufungsbegründungsschrift mit einem auf $\S 823$ Abs. 2 BGB i.V. mit $\S 263$ StGB gestützten „Rückforderungsanspruchs" ist - ohne daß es auf Bedenken wegen der Bestimmtheitsanforderung der Aufrechnung ankäme - bereits deshalb unzulässig, weil ihr das wirksame Aufrechnungsverbot in $\S 3$ des Leasingvertrages entgegensteht. ..."

(Eingesandt vom 5. Zivilsenat des OLG in Frankfurt am Main)

\title{
Rechtsnatur des Überlassungsvertrages
}

\section{OLG Hamm, Urteil vom 28. Mai 1986 (10 U 63/84)}

\section{Nichtamtliche Leitsätze}

1. Der Vertrag über die Úberlassung von Standardprogrammen (hier: für Mikrocomputer) ist ein Werklieferungsvertrag über vertretbare Sachen, auf den gemäß $\$ 651$ BGB Kaufvertragsrecht Anwendung findet.

2. Soll laut Vertrag die Einweisung auf Wunsch des Anwenders separat vereinbart werden, handelt es sich bei der Einweisung um eine Nebenpflicht, die an der Fälligkeit des Kaufpreises nichts ändert.

\author{
Paragraphen \\ BGB: $\S 433 ; \S 651$
}

\section{Stichworte}

Einweisung - rechtliche Einordnung; Programme rechtliche Einordnung; Uberlassung von Standardprogrammen - rechtliche Einordnung 
Tatbestand des LG Hagen (23 O 40/83)

„Die Klägerin verlangt von der Beklagten Bezahlung des Kaufpreises für ... Programme ..." zu einem Preis von ca. DM 3000,- je Programm.

„... Hinsichtlich des Einsatzes der Programme ist Vertragsinhalt, daß ,Einführung und Einsatz der Programme dem Lizenznehmer obliegen. Hierbei berät und unterstützt der Lizenzgeber den Lizenznehmer auf Wunsch. Einzelheiten hierzu sind separat zu vereinbaren'....

Die Beklagte ... ist der Ansicht, sie brauche den verabredeten Kaufpreis nicht zu bezahlen, da sie gemäß $\S 326$ BGB vom Kaufvertrag zurückgetreten sei. Denn die Klägerin habe nicht einwandfreie Software geliefert. ..." Die geschuldete Einweisung sei auch noch nicht erbracht worden.

Die Klägerin obsiegte in beiden Instanzen.

\section{Entscheidungsgründe}

„Die Beklagte war nicht znr Wandelung dieses Vertrages berechtigt, weil die gelieferten Programme nicht die vermeintlichen Fehler aufgewiesen hoben. ...

Unzutreffend ist die Auffassung der Beklagten, die Klageforderung sei gemäß $\S 641 \mathrm{BGB}$ gar nicht fälitig gewesen, weil eine Gesamtabnahme i.S. des $\$ 640$ BGB gefehlt habe. Obschon unter Ziff. 6 des Lizenzvertrages die Gewährleistungsregelung auf $\$ 633 \mathrm{f}$ BGB Bezug nimmt, handelt es sieh bei dem Vertrag zwischen den
Parteien um einen Werklieferungsvertrag über vertretbare Sachen, auf den gemäß $\S 651$ BGB Kaufvertragsrecht Anwendung findet. Die gelieferten Programme sind vertretbare Sachen. Mit dem Zitat des $§ 633$ f BGB mag sich die Klägerin ein Nachbesserungsrecht vorbehalten haben. Es mag auch sein, daß auf vertragliche Nebenpflichten Werkvertragsrecht anzuwenden ist. Es kann aber nicht insgesamt Werkvertragsrecht Anwendung finden, insbesondere nicht $\$ 640 \mathrm{BGB}$, der die Abnahme regelt. In der Auftragsbestätigung wird eine Abnahme gar nicht erwähnt; es heißt dort vielınehr: ,Zahlbar: rein netto bei Anlieferung der Software.'

Zutreffend hat das Landgericht die Unterstützung des Lizenznehmers bei der Einführung und Einsatz der Progtamme durch den Lizenzgeber als Nebenpflicht eingeordnet, die an der Fälligkeit des Preises für die Software nichts ändert."

\section{Anmerkung}

Das Urteil bestätigt die Rechtsprechung, den Vertrag über die Überlassung von Standardprogrammen hinstchtlich der Gewährleistung nach Kaufrecht zu beurteilen (siehe Zahrnt, Gewährleistung bei Überlassung von Standardpregrammen, IuR 1986, 252 ff). Das Urteil bestätigt auch die Auffassung des Rezensenten (Umfang des Einsatzrechts des Anwenders und Programmschutz, IuR 1986, 199 ff), daß hier ein Lizenzvertrag vorliegt.

(ch. z.)

\section{Upgrade-Sonderpreis}

\section{LG Berlin, Urteil vom 26. Februar 1986 (97 O 400/84)}

\section{Nichtamtliche Leitsätze}

1. Ein DV-Programm ist als Ware im Sinne von $\$ 1$ Anordnung des Reichswirtschaftsministers betreffend Sonderveranstaltungen vom 4, 7, 1935 anzusehen.

2. Das befristete Angebot des Erwerbs einer neuen Version eines Standardprogramms gegen einen Sonderpreis stellt eine unerlaubte Sonderveranstaltung dar.

\section{Paragraphen}

Anordnung des Reichswirtschaftsministers betreffend Sonderveranstaltungen vom $4,7,1935: 81$

UWG: $\$ 1, \$ 9 a$

\section{Stichworte}

WettbewerbsverstoB - befristeter Preis für eine neue Version als Sonderveranstaltung

\section{Tatbestand}

Die Beklagte, eine Anbieterin von Mikrocomputerprogrammen, warb mit einem „befristeten Upgrade-Son- derpreis" für ein Standardprogramm. Der Kläger, ein (echter) Wettbewerbsverein hielt das für eine unzulässige Sonderveranstaltung. Er klagte erfolgreich auf Unterlassung.

\section{Entscheidungsgründe}

„Die beanstandete Werbung der Beklagten verstößt gegen $\S \S 1,9$ a UWG, $\S \S 1$ Abs. 1, 2 Abs. 1 der Anordnung des Reichswirtschaftsministers betreffend Sonterveranstaltungen vom 4. 7. 1935. Nach $\S 2$ Abs. 1 der Anordnung ist die Abhaltung von Sonderveranstaltungen im Sinne des $\S 1$ Abs. der Anordnung verbeten. Die Voraussetzungen des $\S 1$ Abs. 2 sind vorliegend erfüllt. Die Beklagte hat für eine Verkaufsveranstaltung geworben. Es liegt nicht lediglich eine Umtauschaktion vor. Die Beklagte will durch die Werbung den Absatz Ihres Computer-Programmes gegen Rückgabe des Programmes ,XXX II' zur Verfügung, sondern verlangt für den Erwerb außerdem einen Preis von 575,- DM zuzüglich Mehrwertsteuer. Die Werbung ist also auf den entgeltlichen Absatz des Programmes gerichtet, wobei Bezieher des Programmes ,XXX III' allerdings wohl den Vorteil haben, daß der Erwerb des Programmes für 